\title{
Recent Applications of the Theory of Lie Systems in Ermakov Systems ${ }^{\star}$
}

\author{
José F. CARIÑENA, Javier DE LUCAS and Manuel F. RAÑADA \\ Department of Theoretical Physics, University of Zaragoza, 50.009 Zaragoza, Spain \\ E-mail: jfc@unizar.es,dlucas@unizar.es,mfran@unizar.es
}

Received November 02, 2007, in final form February 04, 2008; Published online March 12, 2008

Original article is available at http://www.emis.de/journals/SIGMA/2008/031/

\begin{abstract}
We review some recent results of the theory of Lie systems in order to apply such results to study Ermakov systems. The fundamental properties of Ermakov systems, i.e. their superposition rules, the Lewis-Ermakov invariants, etc., are found from this new perspective. We also obtain new results, such as a new superposition rule for the Pinney equation in terms of three solutions of a related Riccati equation.
\end{abstract}

Key words: superposition rule; Pinney equation; Ermakov systems

2000 Mathematics Subject Classification: 34A26; 34A05

\section{Introduction}

Nonlinear equations have been of an increasing interest in Physics during the last thirty years and one of the simplest examples is the today called Milne-Pinney equation

$$
\ddot{x}=-\omega^{2}(t) x+\frac{k}{x^{3}},
$$

where $k \mathrm{a}$ is real constant with values depending on the field in which the equation is to be applied. Such equation was introduced by an Ukrainian mathematician of the nineteenth century, V.P. Ermakov, as a way of looking for a first integral for the time-dependent harmonic oscillator [1]. He was using some of Lie's ideas for dealing with ordinary differential equations with tools of classical geometry. Lie had obtained a characterisation of non-autonomous systems of first-order differential equations

$$
\frac{d x^{i}}{d t}=Y^{i}(t, x), \quad i=1, \ldots, n,
$$

admitting a superposition rule [2]. Such a problem has being receiving these last years very much attention because of its very important applications in physics and mathematics, see [3, $4,5,6,7,8,9,10,11,12,13,14,15,16,17]$. This approach has recently been revisited from a more geometric perspective in [18] where the rôle of the superposition function is played by an appropriate connection. This new approach allows us to consider a superposition of solutions of a given system in order to obtain solutions of another system as it was done in [19]. Our aim in this paper is to show how such a superposition rule may be understood from a geometric viewpoint in some interesting cases, as the Milne-Pinney equation (1) [20, 21], the Ermakov system and its generalisations.

Recall that Ermakov systems are systems of second-order differential equations composed by the Milne-Pinney differential equation (1) together with the corresponding time-dependent

${ }^{\star}$ This paper is a contribution to the Proceedings of the Seventh International Conference "Symmetry in Nonlinear Mathematical Physics" (June 24-30, 2007, Kyiv, Ukraine). The full collection is available at http://www.emis.de/journals/SIGMA/symmetry2007.html 
harmonic oscillator. They have received lot of attention since its introduction in Ermakov's paper [1], where many of its important properties have been exhibited. For instance, he found the time-dependent invariant called Ermakov-Lewis invariant. In a short paper Pinney [21] was able to prove the fundamental result that the general solution of the Milne-Pinney equation can be described in terms of an arbitrary pair of independent particular solutions of the corresponding time-dependent harmonic oscillator and two constants.

Ermakov systems have also been broadly studied in Physics since its introduction until now. They appear in the study of the Bose-Einstein condensates and cosmological models [22, 23, 24], and in the solution of time-dependent harmonic or anharmonic oscillators [25, 26, 27, 28, 29]. Much work has also been devoted to use Hamiltonian or Lagrangian structures in the study of such a system, see e.g. [30, 31], and many generalisations or new insights from the mathematical point of view can be found in [32, 33, 34, 35, 36, 37, 38, 39, 40].

Our aim in this paper is to review the mathematical insight developed in [6] in order to study the properties of Ermakov systems. With this objective, we review in Section 2 the concept of superposition rule, a map describing the general solution of a certain kind of system of differential equations called Lie systems, in terms of a finite set of particular solutions of it. The concept is generalised in Section 3 to express the general solution of a system of second-order differential equations (hereafter shortened as SODE) in terms of arbitrary particular solutions of a new Lie system and some constants. We prove that the theory developed by Lie can easily be adapted to deal with many examples of such SODE systems, and in this way the superposition rules which are generally used for first-order differential equations can also be used for dealing with Ermakov systems. We find room in this way for implicit nonlinear superposition rules in the terminology of [36, 41]. The Ermakov-Lewis invariants [29] appear in a natural way as functions defining the foliation associated to the superposition rule. Some simple examples are used to illustrate the theory. Section 4 is devoted to describe the possibility of a superposition of solutions of a system to give a solution of another related one, and we show how Pinney's results [21] fit in this framework, i.e. we recover the known superposition rule in which the solutions for the Pinney equation are expressed in terms of two solutions of the corresponding $t$-dependent harmonic oscillator and as a straightforward application of our method we show that the general solution of the Pinney equation can also be written as a superposition of three particular solutions of the Riccati equation.

\section{Systems of differential equations admitting a superposition rule}

A superposition rule for solutions of (2) is determined by a function $\Phi: \mathbb{R}^{n(m+1)} \rightarrow \mathbb{R}^{n}$,

$$
x=\Phi\left(x_{(1)}, \ldots, x_{(m)} ; k_{1}, \ldots, k_{n}\right), \quad x_{(a)} \in \mathbb{R}^{n}, \quad a=1, \ldots, m,
$$

such that the general solution can be written, for sufficiently small $t$, as

$$
x(t)=\Phi\left(x_{(1)}(t), \ldots, x_{(m)}(t) ; k_{1}, \ldots, k_{n}\right),
$$

where $\left\{x_{(a)}(t) \mid a=1, \ldots, m\right\}$ is a fundamental set of particular solutions of the system (2) and $k=\left(k_{1}, \ldots, k_{n}\right)$ is a set of $n$ arbitrary constants, whose values fix each particular solution.

A superposition rule provides us a method for finding the general solution of (2) in terms of a set of particular solutions.

According to the Implicit Function Theorem, the function $\Phi: \mathbb{R}^{n(m+1)} \rightarrow \mathbb{R}^{n}$ can be, at least locally around generic points, inverted, so we can write

$$
k=\Psi\left(x_{(0)}, \ldots, x_{(m)}\right)
$$


for a certain function $\Psi: \mathbb{R}^{n(m+1)} \rightarrow \mathbb{R}^{n}$. Hereafter in order to handle a shorter notation we started writing $x_{(0)}$ instead of $x$. The level sets of the superposition function $\Psi$ define a foliation that is invariant under permutations of the $(m+1)$ variables. The functions $\Phi$ and $\Psi$ are related by:

$$
k=\Psi\left(\Phi\left(x_{(1)}, \ldots, x_{(m)} ; k_{1}, \ldots, k_{n}\right), x_{(1)}, \ldots, x_{(m)}\right) .
$$

The fundamental property of the superposition function $\Psi$ is that as

$$
k=\Psi\left(x_{(0)}(t), x_{(1)}(t), \ldots, x_{(m)}(t)\right),
$$

the function $\Psi\left(x_{(0)}, \ldots, x_{(m)}\right)$ is constant along any $(m+1)$-tuple of solutions of the system (2). This implies that if the 'diagonal prolongation' $\widetilde{Y}\left(t, x_{(0)}, \ldots, x_{(m)}\right)$ of the $t$-dependent vector field $Y(t, x)=Y^{i}(t, x) \partial / \partial x^{i}$ is defined by

$$
\tilde{Y}\left(t, x_{(0)}, \ldots, x_{(m)}\right)=\sum_{a=0}^{m} Y_{a}\left(t, x_{(a)}\right), \quad t \in \mathbb{R},
$$

where

$$
Y_{a}\left(t, x_{(a)}\right)=\sum_{i=1}^{n} Y^{i}\left(t, x_{(a)}\right) \frac{\partial}{\partial x_{(a)}^{i}},
$$

then $\widetilde{Y}\left(t, x_{(0)}, \ldots, x_{(m)}\right)$ is a $t$-dependent vector field on $\mathbb{R}^{n(m+1)}$ tangent to the level sets of $\Psi$, i.e. the components $\Psi^{i}$ are constants of motion. The level sets of $\Psi$ corresponding to regular values define a $n$-codimensional foliation $\mathcal{F}$ on an open dense subset $U \subset \mathbb{R}^{n(m+1)}$ and the family $\{\tilde{Y}(t, \cdot), t \in \mathbb{R}\}$ of vector fields in $\mathbb{R}^{n(m+1)}$ consists of vector fields tangent to the leaves of this foliation.

Remark that, as pointed out in [18], for any $k=\left(k_{1}, \ldots, k_{n}\right) \in \mathbb{R}^{n}$ and each $\left(x_{(1)}, \ldots, x_{(m)}\right) \in$ $\mathbb{R}^{n m}$ there is one point $\left(x_{(0)}, x_{(1)}, \ldots, x_{(m)}\right)$ on the level set $\mathcal{F}_{k}$ of the foliation $\mathcal{F}$, namely, such that $\left(\Phi\left(x_{(1)}, \ldots, x_{(m)} ; k\right), x_{(1)}, \ldots, x_{(m)}\right) \in \mathcal{F}_{k}$ (cf. (3)); then, the projection onto the last $m$ factors

$$
\operatorname{pr}:\left(x_{(0)}, x_{(1)}, \ldots, x_{(m)}\right) \in \mathbb{R}^{n(m+1)} \mapsto\left(x_{(1)}, \ldots, x_{(m)}\right) \in \mathbb{R}^{n m}
$$

induces diffeomorphisms on the leaves $\mathcal{F}_{k}$ of $\mathcal{F}$. Such a foliation gives us the superposition principle without referring to the function $\Psi$ : if we fix the point $x_{(0)}(0)$ and $m$ solutions $x_{(1)}(t), \ldots, x_{(m)}(t)$, then $x_{(0)}(t)$ is the unique point in $\mathbb{R}^{n}$ such that $\left(x_{(0)}(t), x_{(1)}(t), \ldots, x_{(m)}(t)\right)$ belongs to the same leaf of $\mathcal{F}$ as $\left(x_{(0)}(0), x_{(1)}(0), \ldots, x_{(m)}(0)\right)$. This means that only $\mathcal{F}$ really matters for the superposition rule.

Lie's main result [2] can be expressed as follows:

Lie's Theorem. The system (2) on a differentiable manifold $N$ admits a superposition rule if and only if the $t$-dependent vector field $Y(t, x)$ can be locally written in the form

$$
Y(t, x)=\sum_{\alpha=1}^{r} b_{\alpha}(t) X_{\alpha}(x)
$$

where the vector fields $X_{\alpha}$, with $\alpha=1, \ldots, r$, close on a $r$-dimensional real Lie algebra, i.e. there exist $r^{3}$ real numbers $c_{\alpha \beta}{ }^{\gamma}$ such that

$$
\left[X_{\alpha}, X_{\beta}\right]=\sum_{\gamma=1}^{r} c_{\alpha \beta}{ }^{\gamma} X_{\gamma}, \quad \forall \alpha, \beta=1, \ldots, r .
$$

In this case we say that (2) is a Lie system. 
The number $m$ of solutions involved in the superposition rule for the Lie system defined by (4) with generic $b_{\alpha}(t)$ is the minimal $k$ such that the diagonal prolongations of $X_{1}, \ldots, X_{r}$ to $N^{k}, \widetilde{X}_{1}, \ldots, \widetilde{X}_{r}$, are linearly independent at (generically) each point: for $k=m$ the only real numbers solution of the linear system

$$
\sum_{\alpha=1}^{r} c_{\alpha} \widetilde{X}_{\alpha}\left(x_{(1)}, \ldots, x_{(k)}\right)=0,
$$

at a generic point $\left(x_{(1)}, \ldots, x_{(k)}\right)$ is the trivial solution $c_{\alpha}=0$, with $\alpha=1, \ldots, r$, and there are nontrivial solutions for $k<m$. Then, the superposition function $\Psi$ is made up of $t$-independent constants of motion for the prolonged $t$-dependent vector field $\widetilde{Y}$. We shall call them firstintegrals.

A possible generalisation consists on considering foliations of codimension different from $n$, or even choosing different Lie systems with the same associated Lie algebra for defining the prolonged vector field. These facts will be illustrated with several examples in the following sections.

\section{SODE Lie systems}

A system of second-order differential equations

$$
\frac{d^{2} x^{i}}{d t^{2}}=F^{i}\left(t, x, \frac{d x}{d t}\right), \quad i=1, \ldots, n
$$

can be studied through the system of first-order differential equations

$$
\begin{aligned}
& \dot{x}^{i}=v^{i}, \\
& \dot{v}^{i}=F^{i}(t, x, v), \quad i=1, \ldots, n,
\end{aligned}
$$

with an associated $t$-dependent vector field

$$
X=v^{i} \frac{\partial}{\partial x^{i}}+F^{i}(t, x, v) \frac{\partial}{\partial v^{i}} .
$$

We call SODE Lie systems those SODE for which $X$ is a Lie system, i.e. $X$ can be written as a linear combination with $t$-dependent coefficients of vector fields closing a finite-dimensional real Lie algebra. Even if we are focusing here our attention on second-order differential equations, the approach can be straightforwardly applied for $r$-order differential equations. Some particular examples of second-order Lie systems are given in next subsections.

\subsection{Milne-Pinney equation}

The Milne-Pinney equation (1) is a second-order nonlinear differential equation [20, 21] that describes the time-evolution of an isotonic oscillator $[42,43]$ (also called pseudo-oscillator), i.e. a harmonic oscillator with inverse quadratic potential [32]. This nonlinear oscillator shares with the harmonic one the property of having a period independent of the energy [44], i.e. it is a isochronous system, and its quantum version has an equispaced spectrum $[45,46]$.

Note that if $x(t)$ is solution of $(1)$ then $x^{\prime}(t)=-x(t)$ is solution too and we can restrict ourselves to consider solutions in the half-line $\mathbb{R}_{+}=\{x \in \mathbb{R} \mid x>0\}$. Thus, we are interested in the solutions of (1) that are curves in $\mathbb{R}_{+}$. 
We can relate the Milne-Pinney equation with a system of first-order differential equations defined in $\mathrm{TR}_{+}$by introducing a new auxiliary variable $v \equiv \dot{x}$. Such system is given by

$$
\begin{aligned}
\dot{x} & =v, \\
\dot{v} & =-\omega^{2}(t) x+\frac{k}{x^{3}},
\end{aligned}
$$

where $x \in \mathbb{R}_{+}$and $(x, v) \in \mathrm{T}_{x} \mathbb{R}_{+}$. By the sake of simplicity we assume from now on that $k>0$ but similar results can be obtained for $k<0$. Now, the associated $t$-dependent vector field over $\mathrm{TR}_{+}$is

$$
X=v \frac{\partial}{\partial x}+\left(-\omega^{2}(t) x+\frac{k}{x^{3}}\right) \frac{\partial}{\partial v} .
$$

This is a Lie system because $X$ can be written as

$$
X=L_{2}-\omega^{2}(t) L_{1},
$$

where the vector fields $L_{1}$ and $L_{2}$ are given by

$$
L_{1}=x \frac{\partial}{\partial v}, \quad L_{2}=v \frac{\partial}{\partial x}+\frac{k}{x^{3}} \frac{\partial}{\partial v},
$$

which are such that

$$
\left[L_{1}, L_{2}\right]=2 L_{3}, \quad\left[L_{3}, L_{2}\right]=-L_{2}, \quad\left[L_{3}, L_{1}\right]=L_{1}
$$

with

$$
L_{3}=\frac{1}{2}\left(x \frac{\partial}{\partial x}-v \frac{\partial}{\partial v}\right),
$$

i.e. they span a 3 -dimensional real Lie algebra $\mathfrak{g}$ isomorphic to $\mathfrak{s l}(2, \mathbb{R})$. Another different relation of the Lie algebra $\mathfrak{s l}(2, \mathbb{R})$ with this system has been pointed out but in the context of Lie symmetries $[47,48]$. On the contrary here $\mathfrak{s l}(2, \mathbb{R})$ is the Lie algebra of vector fields of the Lie system and the search for the general solution of (6) can be reduced to finding the solution starting from the neutral element of the equation in the group $S L(2, \mathbb{R})$ given by $\dot{g} g^{-1}=\omega^{2}(t) \mathrm{a}_{1}-\mathrm{a}_{2}($ see $[5,6])$, where $\left\{\mathrm{a}_{1}, \mathrm{a}_{2}, \mathrm{a}_{3}\right\}$ is the following basis of $\mathfrak{s l}(2, \mathbb{R})$ :

$$
\mathrm{a}_{1}=\left(\begin{array}{cc}
0 & 0 \\
-1 & 0
\end{array}\right), \quad \mathrm{a}_{2}=\left(\begin{array}{cc}
0 & -1 \\
0 & 0
\end{array}\right), \quad \mathrm{a}_{3}=\frac{1}{2}\left(\begin{array}{cc}
-1 & 0 \\
0 & 1
\end{array}\right),
$$

which satisfy the same commutation relations as the vector fields $\left\{L_{j} \mid j=1,2,3\right\}$. Actually, it is possible to show that $L_{j}$ is the fundamental vector field corresponding to $\mathrm{a}_{j}$ with respect to the action $\Phi:(A,(x, v)) \in S L(2, \mathbb{R}) \times \mathrm{TR}_{+} \rightarrow(\bar{x}, \bar{v}) \in \mathrm{TR}_{+}$given by:

$$
\begin{aligned}
& \bar{x}=\sqrt{\frac{k+\left[(\beta v+\alpha x)(\delta v+\gamma x)+k\left(\delta \beta / x^{2}\right)\right]^{2}}{(\delta v+\gamma x)^{2}+k(\delta / x)^{2}}}, \\
& \bar{v}=\kappa \sqrt{(\delta v+\gamma x)^{2}+\frac{k \delta^{2}}{x^{2}}\left(1-\frac{x^{2}}{\delta^{2} \bar{x}^{2}}\right)} \quad \text { if } \quad A \equiv\left(\begin{array}{cc}
\alpha & \beta \\
\gamma & \delta
\end{array}\right),
\end{aligned}
$$

where $\kappa$ is \pm 1 or 0 depending on the initial point $(x, v)$ and the element of the group $S L(2, \mathbb{R})$ that acts on it. In order to obtain an explicit expression for $\kappa$ in terms of $A$ and $(x, v)$ we can use the following decomposition for any element of the group $S L(2, \mathbb{R})$

$$
A=\exp \left(-\alpha_{2} \mathrm{a}_{2}\right) \exp \left(-\alpha_{1} \mathrm{a}_{1}\right) \exp \left(-\alpha_{3} \mathrm{a}_{3}\right)=\left(\begin{array}{cc}
1 & \alpha_{2} \\
0 & 1
\end{array}\right)\left(\begin{array}{cc}
1 & 0 \\
\alpha_{1} & 1
\end{array}\right)\left(\begin{array}{cc}
e^{\alpha_{3} / 2} & 0 \\
0 & e^{-\alpha_{3} / 2}
\end{array}\right)
$$


from where we obtain that $\alpha_{1}=\gamma \delta$ and $\alpha_{2}=\beta / \delta$. As we know that $\Phi\left(\exp \left(-\alpha_{3} \mathrm{a}_{3}\right),(x, v)\right)$ is the integral curve of the vector field $L_{3}$ starting from the point $(x, v)$ parametrised by $\alpha_{3}$, it is straightforward to check that

$$
\left(x_{1}, v_{1}\right) \equiv \Phi\left(\exp \left(-\alpha_{3} \mathrm{a}_{3}\right),(x, v)\right)=\left(\exp \left(\alpha_{3} / 2\right) x, \exp \left(-\alpha_{3} / 2\right) v\right),
$$

and in a similar way

$$
\left(x_{2}, v_{2}\right) \equiv \Phi\left(\exp \left(-\alpha_{1} \mathrm{a}_{1}\right),\left(x_{1}, v_{1}\right)\right)=\left(x_{1}, \alpha_{1} x_{1}+v_{1}\right) .
$$

Finally, we want to obtain $(\bar{x}, \bar{v})=\Phi\left(\exp \left(-\alpha_{2} \mathrm{a}_{2}\right),\left(x_{2}, v_{2}\right)\right)$, and taking into account that the integral curves of $L_{2}$ satisfy that

$$
\frac{x^{3} d v}{k}=\frac{d x}{v}=d \alpha_{2},
$$

it turns out that when $k>0$ we have $\bar{v}^{2}+k / \bar{x}^{2}=v_{2}^{2}+k / x_{2}^{2} \equiv \lambda$ with $\lambda>0$. Thus, using this fact and (8) we obtain

$$
\frac{k^{1 / 2} d v}{\left(\lambda-v^{2}\right)^{3 / 2}}=d \alpha_{2}
$$

and integrating $v$ between $v_{2}$ and $\bar{v}$,

$$
\frac{\bar{v}}{\left(\lambda-\bar{v}^{2}\right)^{1 / 2}}=\alpha_{2} \frac{\lambda}{k^{1 / 2}}+\frac{v_{2}}{\left(\lambda-v_{2}^{2}\right)^{1 / 2}}=\frac{1}{k^{1 / 2}}\left(\alpha_{2} \lambda+v_{2}\left|x_{2}\right|\right) .
$$

As $\kappa=\operatorname{sign}[\bar{v}]$, we see that $\kappa$ is given by

$$
\kappa=\operatorname{sign}\left[\alpha_{2} \lambda+v_{2}\left|x_{2}\right|\right]=\operatorname{sign}\left[\frac{\beta}{\delta}(x \gamma+v \delta)^{2}+\frac{k \delta \beta}{x^{2}}+\frac{|x|}{\delta}(v \delta+x \gamma)\right] .
$$

Non-trivial first-integrals independent of $\omega(t)$ for the equation (1) do not exist, i.e. there is not any $t$-independent constant of motion $I: U \subset \mathrm{T} \mathbb{R}_{+} \rightarrow \mathbb{R}$ such that $X I=0$ for any function $\omega(t)$. This is equivalent to $d I\left(L_{j}\right)=0$ for $L_{j}$ with $j=1,2,3$. Thus, the integrals of motion we are looking for verify that $d I$ vanishes on the involutive distribution $\mathcal{V}(x) \simeq\left\langle L_{1}(x), L_{2}(x), L_{3}(x)\right\rangle$ generated by the fundamental vector fields $L_{j}$. In almost any point we have that $\mathcal{V}(x)=\mathrm{T}_{x} \mathrm{TR}_{+}$, and as $d I=0$ on dense subsets in $T \mathbb{R}_{+}$, the only possibility is $d I=0$, therefore $I$ is a constant, and the integral of motion is trivial.

\subsection{Generalised Ermakov system}

Consider a possible generalisations of the Ermakov system given by

$$
\begin{aligned}
& \ddot{x}=-\omega^{2}(t) x+\frac{1}{x^{3}} f(y / x), \\
& \ddot{y}=-\omega^{2}(t) y+\frac{1}{y^{3}} g(y / x) .
\end{aligned}
$$

This system of differential equations has been broadly studied in [27, 30, 35, 37, 41, 49, 50]. In this section we analyse how can we make use of the theory of Lie systems in order to obtain the known first-integral of motion for this system of differential equations.

Note that if $(x(t), y(t))$ is a solution, then $(-x(t),-y(t))$ is also a solution for $(9)$, and therefore we can focus our attention on the solutions of generalised Ermakov system which are curves in the manifold $\mathbb{R}_{+} \times \mathbb{R}$. 
The generalised Ermakov system (9) can be written as a system of first-order differential equations with a double number of degrees of freedom by introducing two new variables $v_{x}$ and $v_{y}$,

$$
\begin{aligned}
& \dot{x}=v_{x}, \\
& \dot{v}_{x}=-\omega^{2}(t) x+\frac{1}{x^{3}} f(y / x), \\
& \dot{y}=v_{y}, \\
& \dot{v}_{y}=-\omega^{2}(t) y+\frac{1}{y^{3}} g(y / x),
\end{aligned}
$$

and its solutions can be studied from the integral curves of the $t$-dependent vector field $X$ in $\mathrm{T}\left(\mathbb{R}_{+} \times \mathbb{R}\right)$ given by

$$
X=v_{x} \frac{\partial}{\partial x}+v_{y} \frac{\partial}{\partial y}+\left(-\omega^{2}(t) x+\frac{1}{x^{3}} f(y / x)\right) \frac{\partial}{\partial v_{x}}+\left(-\omega^{2}(t) y+\frac{1}{y^{3}} g(y / x)\right) \frac{\partial}{\partial v_{y}},
$$

which can be written as a linear combination

$$
X=N_{2}-\omega^{2}(t) N_{1},
$$

where $N_{1}$ and $N_{2}$ are the vector fields

$$
N_{1}=x \frac{\partial}{\partial v_{x}}+y \frac{\partial}{\partial v_{y}}, \quad N_{2}=v_{x} \frac{\partial}{\partial x}+v_{y} \frac{\partial}{\partial y}+\frac{1}{x^{3}} f(y / x) \frac{\partial}{\partial v_{x}}+\frac{1}{y^{3}} g(y / x) \frac{\partial}{\partial v_{y}} .
$$

Note that these vector fields generate a 3-dimensional real Lie algebra with a third generator

$$
N_{3}=\frac{1}{2}\left(x \frac{\partial}{\partial x}+y \frac{\partial}{\partial y}-v_{x} \frac{\partial}{\partial v_{x}}-v_{y} \frac{\partial}{\partial v_{y}}\right) .
$$

In fact, as

$$
\left[N_{1}, N_{2}\right]=2 N_{3}, \quad\left[N_{3}, N_{1}\right]=N_{1}, \quad\left[N_{2}, N_{3}\right]=N_{2},
$$

they generate a Lie algebra isomorphic to $\mathfrak{s l}(2, \mathbb{R})$. Therefore the generalised Ermakov system is a SODE Lie system with associated Lie algebra $\mathfrak{s t}(2, \mathbb{R})$.

The integrable distribution associated to the Lie system (10) is of dimension three, while the manifold $\mathrm{T}\left(\mathbb{R}_{+} \times \mathbb{R}\right)$ is 4-dimensional, and then there exists a first-integral of motion, $F: \mathbb{R}^{4} \rightarrow \mathbb{R}$, for any $\omega^{2}(t)$. This first-integral $F$ satisfies $N_{i} F=0$ for $i=1,2,3$, but as $\left[N_{1}, N_{2}\right]=2 N_{3}$ it is enough to impose $N_{1} F=N_{2} F=0$. Then, if $N_{1} F=0$, by the method of characteristics we can conclude that there exists a function $\bar{F}: \mathbb{R}^{3} \rightarrow \mathbb{R}$ such that $F\left(x, y, v_{x}, v_{y}\right)=\bar{F}(x, y, \xi)$ with $\left.\xi=x v_{y}-y v_{x}\right)$. The condition $N_{2} F=0$ reads now

$$
v_{x} \frac{\partial \bar{F}}{\partial x}+v_{y} \frac{\partial \bar{F}}{\partial y}+\left(-\frac{y}{x^{3}} f(y / x)+\frac{x}{y^{3}} g(y / x)\right) \frac{\partial \bar{F}}{\partial \xi}=0 .
$$

We can therefore consider the associated system of the characteristics:

$$
\frac{d x}{v_{x}}=\frac{d y}{v_{y}}=\frac{d \xi}{-\frac{y}{x^{3}} f(y / x)+\frac{x}{y^{3}} g(y / x)}
$$

and using that

$$
\frac{-y d x+x d y}{\xi}=\frac{d x}{v_{x}}=\frac{d y}{v_{y}},
$$


we arrive to

$$
\frac{-y d x+x d y}{\xi}=\frac{d \xi}{-\frac{y}{x^{3}} f\left(\frac{y}{x}\right)+\frac{x}{y^{3}} g\left(\frac{y}{x}\right)},
$$

i.e.

$$
-\frac{y^{2} d\left(\frac{x}{y}\right)}{\xi}=\frac{d \xi}{-\frac{y}{x^{3}} f\left(\frac{y}{x}\right)+\frac{x}{y^{3}} g\left(\frac{y}{x}\right)}
$$

and integrating we obtain the following first-integral

$$
\frac{1}{2} \xi^{2}+\int^{x / y}\left[-\frac{1}{\zeta^{3}} f\left(\frac{1}{\zeta}\right)+\zeta g\left(\frac{1}{\zeta}\right)\right] d \zeta=C .
$$

This first-integral allows us to determine, by means of quadratures, a solution of a subsystem in terms of a solution of the other equation.

\subsection{The harmonic oscillator with time-dependent frequency}

Our aim in this section is to exhibit with a simple example that we can define new Lie systems of differential equations admitting first-integrals of motion which do not depend on the timedependent coefficients by putting together some copies of the same Lie system in the way indicated in Section 2. These integrals of motion allow us to obtain relations between different solutions of the initial Lie systems and can be used to construct a superposition rule if enough copies have been added.

The equation of motion of a 1-dimensional harmonic oscillator with time-dependent frequency is $\ddot{x}=-\omega^{2}(t) x$, which is a second-order differential equation whose solutions are curves in $\mathbb{R}$. We can alternatively consider the following system of first-order differential equations

$$
\begin{aligned}
& \dot{x}=v, \\
& \dot{v}=-\omega^{2}(t) x,
\end{aligned}
$$

whose solutions are the integral curves in $T \mathbb{R}$ of the $t$-dependent vector field

$$
X=v \frac{\partial}{\partial x}-\omega^{2}(t) x \frac{\partial}{\partial v} .
$$

Now $X$ is a linear combination $X=X_{2}-\omega^{2}(t) X_{1}$, where $X_{1}$ and $X_{2}$ are vector fields in $\mathrm{TR}$ :

$$
X_{1}=x \frac{\partial}{\partial v}, \quad X_{2}=v \frac{\partial}{\partial x}
$$

such that

$$
\left[X_{1}, X_{2}\right]=2 X_{3}, \quad\left[X_{3}, X_{1}\right]=X_{1}, \quad\left[X_{2}, X_{3}\right]=X_{2},
$$

where $X_{3}$ is the vector field given by

$$
X_{3}=\frac{1}{2}\left(x \frac{\partial}{\partial x}-v \frac{\partial}{\partial v}\right)
$$

As a consequence of Lie's Theorem we see that $X$ defines a Lie system with associated Lie algebra $\mathfrak{s l}(2, \mathbb{R})$. Actually, the vector fields $\left\{X_{\alpha} \mid \alpha=1,2,3\right\}$ are fundamental vector fields corresponding to the usual linear action of $S L(2, \mathbb{R})$ on $\mathbb{R}^{2} \simeq \mathrm{TR}$ given by

$$
\bar{x}=\alpha x+\beta v, \quad \bar{v}=\gamma x+\delta v \quad \text { if } \quad A \equiv\left(\begin{array}{cc}
\alpha & \beta \\
\gamma & \delta
\end{array}\right) .
$$


Remark that the elements of the basis of $\mathfrak{s l}(2, \mathbb{R})$ given by $(7)$ close the same commutation relations as the vector fields $\left\{X_{\alpha} \mid \alpha=1,2,3\right\}$ which turn out to be the associated fundamental vector fields for the elements $\mathrm{a}_{\alpha}$.

Non-trivial first-integrals independent of $\omega(t)$ for the system (11) do not exist, because the fundamental vector fields generate the involutive distribution $\mathcal{V} \simeq\left\langle X_{1}, X_{2}, X_{3}\right\rangle$ whose values in each point of $T \mathbb{R}$ generate the corresponding tangent space in almost any point and then as $d I$ vanishes on $\mathcal{V}$, therefore $d I$ vanishes in a generic point. Thus $I$ is a trivial first integral of motion.

As it was said before, non-trivial integrals of motion are needed to develop the procedures of integration indicated in [6], i.e. we should add copies of the same differential equation until the linear subspace of the involutive distribution of the total system in a generic point has a dimension smaller than the dimension of the manifold. In this way there is room for a nonconstant function $I$ such that $d I$ vanishes on the subspace of the distribution associated with the extended system of differential equations.

Thus, we first consider a system with two copies of the same differential equation,

$$
\begin{aligned}
& \ddot{x}_{1}=-\omega^{2}(t) x_{1}, \\
& \ddot{x}_{2}=-\omega^{2}(t) x_{2},
\end{aligned}
$$

which corresponds to a 2-dimensional isotropic harmonic oscillator with a time-dependent frequency and is associated with the following system of first-order differential equations

$$
\begin{aligned}
& \dot{x}_{1}=v_{1}, \\
& \dot{v}_{1}=-\omega^{2}(t) x_{1}, \\
& \dot{x}_{2}=v_{2}, \\
& \dot{v}_{2}=-\omega^{2}(t) x_{2},
\end{aligned}
$$

whose solutions are the integral curves of the $t$-dependent vector field

$$
X=v_{1} \frac{\partial}{\partial x_{1}}+v_{2} \frac{\partial}{\partial x_{2}}-\omega^{2}(t) x_{1} \frac{\partial}{\partial v_{1}}-\omega^{2}(t) x_{2} \frac{\partial}{\partial v_{2}},
$$

which is a linear combination, $X=X_{2}-\omega^{2}(t) X_{1}$, with $X_{1}$ and $X_{2}$ being the vector fields

$$
X_{1}=x_{1} \frac{\partial}{\partial v_{1}}+x_{2} \frac{\partial}{\partial v_{2}}, \quad X_{2}=v_{1} \frac{\partial}{\partial x_{1}}+v_{2} \frac{\partial}{\partial x_{2}},
$$

which are such that

$$
\left[X_{1}, X_{2}\right]=2 X_{3}, \quad\left[X_{3}, X_{1}\right]=X_{1}, \quad\left[X_{2}, X_{3}\right]=X_{2},
$$

where the vector field $X_{3}$ is defined by

$$
X_{3}=\frac{1}{2}\left(x_{1} \frac{\partial}{\partial x_{1}}+x_{2} \frac{\partial}{\partial x_{2}}-v_{1} \frac{\partial}{\partial v_{1}}-v_{2} \frac{\partial}{\partial v_{2}}\right) .
$$

The system of differential equations (14) is therefore a Lie system, i.e. the $t$-dependent vector field can be written as a linear combination with time-dependent coefficients of vector fields $X_{\alpha}$ closing a finite dimensional Lie algebra. Such vector fields are diagonal prolongations of the fundamental vector fields of the initial system of differential equation and satisfy the same commutation relations (15) as (12). The subspace of the distribution associated for this Lie system has a rank lower or equal to the dimension of the Lie algebra. The Lie algebra associated with the $t$-dependent vector field $X$ is also $\mathfrak{s l}(2, \mathbb{R})$. 
Note that the system (11) did not admit non-trivial first-integral of motion independent of $\omega(t)$, but the system (13) does admit a first-integral because the subspace of the distribution generated by the fundamental vector fields has rank three in almost any point and the dimension of the total manifold is four. Thus, there exists a function $F$ such that $d F$ is vanishes on the distribution generated by the $X_{\alpha}$ 's. The function $F\left(x_{1}, x_{2}, v_{1}, v_{2}\right)$ is such that $X_{1} F=0$ iff there exists a function $\bar{F}\left(x_{1}, x_{2}, \xi\right)$ with $\xi=x_{1} v_{2}-x_{2} v_{1}$, such that $F\left(x_{1}, x_{2}, v_{1}, v_{2}\right)=\bar{F}\left(x_{1}, x_{2}, \xi\right)$, and then the second condition $X_{2} F=0$ implies that $\bar{F}$ can only depend on $\xi$, i.e. $F\left(x_{1}, x_{2}, v_{1}, v_{2}\right)=\hat{F}(\xi)$. From the commutation relation $2 X_{3}=\left[X_{1}, X_{2}\right]$, we see that the conditions $X_{1} F=X_{2} F=0$ imply $X_{3} F=0$, and therefore any first integral is a function of $F\left(x_{1}, x_{2}, v_{1}, v_{2}\right)=x_{1} v_{2}-x_{2} v_{1}$, which physically corresponds to the angular momentum. This first integral can be seen from the mathematical viewpoint as a partial superposition rule [6]. Actually, if $x_{1}(t)$ is a solution of the first equation, then we obtain for each real number $k$ the first-order differential equation for the variable $x_{2}$

$$
x_{1}(t) \frac{d x_{2}}{d t}=k+\dot{x}_{1}(t) x_{2}
$$

from where $x_{2}$ can be found to be given by

$$
x_{2}(t)=k^{\prime} x_{1}(t)+k x_{1}(t) \int^{t} \frac{d \zeta}{x_{1}^{2}(\zeta)},
$$

where $k^{\prime}$ is a new integration constant.

In order to look for a superposition rule we should consider a system of some copies of (11) with at least as many integrals of motion as the dimension of the initial manifold. Then it may be possible to obtain the variables of the initial manifold explicitly in terms of the other variables. Following the development of the Section 2 a way to obtain the number of necessary particular solutions to obtain a superposition rule is to consider a set of copies of the initial system and check out if the prolongations of the vector fields $X_{1}, X_{2}$ and $X_{3}$ are linearly independent in a generic point.

In the case of two copies of the $t$-dependent harmonic oscillator it is possible to show that in a generic point if $\lambda_{1} \tilde{X}_{1}+\lambda_{2} \tilde{X}_{2}+\lambda_{3} \tilde{X}_{3}$ vanishes then $\lambda_{1}=\lambda_{2}=\lambda_{3}=0$, therefore $m=2$ and consequently there is a superposition rule involving two particular solutions. In order to obtain the superposition rule we need to consider three copies of the $t$-dependent harmonic oscillator and study the system of first-order differential equations

$$
\begin{aligned}
& \dot{x}_{1}=v_{1}, \\
& \dot{v}_{1}=-\omega^{2}(t) x_{1}, \dot{x}_{2}=v_{2}, \\
& \dot{v}_{2}=-\omega^{2}(t) x_{2}, \\
& \dot{x}=v, \\
& \dot{v}=-\omega^{2}(t) x,
\end{aligned}
$$

whose solutions are the integral curves of the $t$-dependent vector field

$$
X=v_{1} \frac{\partial}{\partial x_{1}}+v_{2} \frac{\partial}{\partial x_{2}}+v \frac{\partial}{\partial x}-\omega^{2}(t) x_{1} \frac{\partial}{\partial v_{1}}-\omega^{2}(t) x_{2} \frac{\partial}{\partial v_{2}}-\omega^{2}(t) x \frac{\partial}{\partial v},
$$

which is a linear combination, $X=X_{2}-\omega^{2}(t) X_{1}$, with $X_{1}$ and $X_{2}$ being the vector fields

$$
X_{1}=x_{1} \frac{\partial}{\partial v_{1}}+x_{2} \frac{\partial}{\partial v_{2}}+x \frac{\partial}{\partial v}, \quad X_{2}=v_{1} \frac{\partial}{\partial x_{1}}+v_{2} \frac{\partial}{\partial x_{2}}+v \frac{\partial}{\partial x},
$$

which are such that

$$
\left[X_{1}, X_{2}\right]=2 X_{3}, \quad\left[X_{3}, X_{1}\right]=X_{1}, \quad\left[X_{2}, X_{3}\right]=X_{2},
$$


where the vector field $X_{3}$ is defined by

$$
X_{3}=\frac{1}{2}\left(x_{1} \frac{\partial}{\partial x_{1}}+x_{2} \frac{\partial}{\partial x_{2}}+x \frac{\partial}{\partial x}-v_{1} \frac{\partial}{\partial v_{1}}-v_{2} \frac{\partial}{\partial v_{2}}-v \frac{\partial}{\partial v}\right) .
$$

The first-integrals $F$ are as in the last case the solutions of $X_{1} F=X_{2} F=0$, because $2 X_{3}=\left[X_{1}, X_{2}\right]$. The condition $X_{1} F=0$ says that there exists a function $\bar{F}: \mathbb{R}^{5} \rightarrow \mathbb{R}^{2}$ such that $F\left(x_{1}, x_{2}, x, v_{1}, v_{2}, v\right)=\bar{F}\left(x_{1}, x_{2}, x, \xi_{1}, \xi_{2}\right)$ with $\xi_{1}\left(x_{1}, x_{2}, x, v_{1}, v_{2}, v\right)=x v_{1}-x_{1} v$ and $\xi_{2}\left(x_{1}, x_{2}, x, v_{1}, v_{2}, v\right)=x v_{2}-x_{2} v$, and when written in terms of $\bar{F}$ the condition $X_{2} F=0$ implies that $\bar{F}$ is an arbitrary function of $\xi_{1}$ and $\xi_{2}$ (of course, $\xi=x_{1} v_{2}-x_{2} v_{1}$ is also a first-integral). They produce a superposition rule, because from

$$
x v_{2}-x_{2} v=k_{1}, \quad x_{1} v-v_{1} x=k_{2}
$$

we obtain the expected superposition rule for two solutions:

$$
x=c_{1} x_{1}+c_{2} x_{2}, \quad v=c_{1} v_{1}+c_{2} v_{2}, \quad c_{i}=\frac{k_{1}}{k}, \quad k=x_{1} v_{2}-x_{2} v_{1} .
$$

\section{Construction of mixed superposition rules}

In the preceding sections we have considered SODE Lie systems together with some copies of the same system. In this section we show a way to deal with sets of maybe different systems of differential equations sharing the same associated Lie algebra as Lie systems. These sets satisfy certain conditions to be explained later on and can be used to obtain mixed superposition rules.

Suppose that a Lie system is given

$$
\frac{d x}{d t}=\sum_{\alpha=1}^{r} b_{\alpha}(t) X_{\alpha}(x),
$$

where the $X_{\alpha}$ with $\alpha=1, \ldots, r$, close a finite-dimensional Lie algebra as given by (5). We want to obtain a mixed superposition rule for this Lie system, i.e. a function $\Phi: N^{1} \times \cdots \times N^{m} \times \mathbb{R}^{n} \rightarrow N$ in such a way that any solution integral curve $x(t)$ of $X(t, \cdot)$ be given by

$$
x(t)=\Phi\left(x_{(1)}(t), \ldots, x_{m}(t), k_{1}, \ldots, k_{n}\right),
$$

where $x_{(1)}(t), \ldots, x_{(m)}(t)$, are integral curves of the $t$-dependent vector fields on the corresponding manifolds $N^{j}$ with $j=1, \ldots, m$ and given by

$$
\begin{aligned}
& X^{(1)}(t, \cdot)=\sum_{\alpha=1}^{r} b_{\alpha}(t) X_{\alpha}^{1}(\cdot), \\
& \ldots \ldots \ldots \ldots \ldots \ldots \ldots \ldots \ldots \ldots
\end{aligned}
$$

and in such a way that $X_{\alpha}^{j}$ with $j=1, \ldots, m$, and $\alpha=1, \ldots, r$, close the same commutation relations as the vector fields $X_{\alpha}$, i.e.

$$
\left[X_{\alpha}^{j}, X_{\beta}^{j}\right]=c_{\alpha \beta}{ }^{\gamma} X_{\gamma}^{j}, \quad j=1, \ldots, m, \quad \text { and } \quad \alpha, \beta=1, \ldots r .
$$

The search for a set of vector fields closing a given Lie algebra in a certain manifold amounts to look for different actions of the corresponding Lie group. 
Then, over $\tilde{N}$ we obtain that

$$
\left[X_{\alpha}, X_{\beta}^{j}\right]=0, \quad\left[X_{\alpha}^{j}, X_{\beta}^{k}\right]=0, \quad j, k=1, \ldots, m, \quad \text { with } \quad j \neq k, \quad \text { and } \quad \alpha, \beta=1, \ldots r,
$$

and because of these commutation relations we can define in $\tilde{N}$ the vector fields

$$
\tilde{Y}_{\alpha}=X_{\alpha}+\sum_{i=1}^{m} X_{\alpha}^{i}
$$

which satisfy the same commutation relations as the vector fields $X_{\alpha}$ in (5). Thus, the system of differential equations determining the integral curves of the $t$-dependent vector field

$$
\tilde{Y}(t, \cdot)=\sum_{\alpha=1}^{r} b_{\alpha}(t) \tilde{Y}_{\alpha}(\cdot)
$$

is a Lie system with the same associated Lie algebra as the initial system of differential equations. Nevertheless, the dimension of the manifold is this time larger than the dimension of $N$ because we have put together several manifolds. The distribution $\tilde{\mathcal{V}}$ associated to this Lie system is given by $\tilde{\mathcal{V}}(\tilde{x})=\left\langle\tilde{Y}_{1}(\tilde{x}), \ldots, \tilde{Y}_{r}(\tilde{x})\right\rangle$ and is involutive. The dimension of the subspace in a point is lower or equal to $r$ and therefore when the dimension of the manifold $\tilde{N}$ is larger than $r$ there are integrals of motion for any value of the time-dependent coefficients which may be used to obtain superposition rules.

In the next section we give some examples of the application of this procedure to the Ermakov system in order to both recover previously known properties and to find also new results.

\subsection{Ermakov system}

Consider the system of ordinary first-order differential equations $[28,40]$

$$
\begin{aligned}
& \dot{x}=v_{x}, \\
& \dot{v}_{x}=-\omega^{2}(t) x, \\
& \dot{y}=v_{y}, \\
& \dot{v}_{y}=-\omega^{2}(t) y+\frac{1}{y^{3}}
\end{aligned}
$$

made up by a 1-dimensional harmonic oscillator and the Milne-Pinney equation with $k=1$, which has the above-mentioned conditions. Its solutions are the integral curves of the $t$-dependent vector field

$$
X=v_{x} \frac{\partial}{\partial x}+v_{y} \frac{\partial}{\partial y}-\omega^{2}(t) x \frac{\partial}{\partial v_{x}}+\left(-\omega^{2}(t) y+\frac{1}{y^{3}}\right) \frac{\partial}{\partial v_{y}},
$$

which is a linear combination with time-dependent coefficients, $X=X_{2}-\omega^{2}(t) X_{1}$, of

$$
X_{1}=x \frac{\partial}{\partial v_{x}}+y \frac{\partial}{\partial v_{y}}, \quad X_{2}=v_{x} \frac{\partial}{\partial x}+v_{y} \frac{\partial}{\partial y}+\frac{1}{y^{3}} \frac{\partial}{\partial v_{y}} .
$$

This system closes on a $\mathfrak{s l}(2, \mathbb{R})$ Lie algebra with $X_{3}$ given by

$$
X_{3}=\frac{1}{2}\left(x \frac{\partial}{\partial x}+y \frac{\partial}{\partial y}-v_{x} \frac{\partial}{\partial v_{x}}-v_{y} \frac{\partial}{\partial v_{y}}\right) .
$$

The generators of this Lie system span a distribution of dimension two and there is no first-integral of the motion for such subsystem. By adding the other $\mathfrak{s l}(2, \mathbb{R})$ linear Lie system 
appearing in the Ermakov system, the harmonic oscillator with the same time-dependent angular frequency, as the rank of the space of the distribution in the 4-dimensional space is three, there is an integral of motion. The first-integral can be obtained from $X_{1} F=X_{2} F=0$. But $X_{1} F=0$ means that there exists a function $\bar{F}: \mathbb{R}^{3} \rightarrow \mathbb{R}$ such that $F\left(x, y, v_{x}, v_{y}\right)=\bar{F}(x, y, \xi)$, with $\xi=x v_{y}-y v_{x}$, and then $X_{2} F=0$ is written

$$
v_{x} \frac{\partial \bar{F}}{\partial x}+v_{y} \frac{\partial \bar{F}}{\partial y}+\frac{x}{y^{3}} \frac{\partial \bar{F}}{\partial \xi}
$$

and we obtain the associated system of characteristics

$$
\frac{x d y-y d x}{\xi}=\frac{y^{3} d \xi}{x} \Longrightarrow \frac{d(x / y)}{\xi}+\frac{y d \xi}{x}=0,
$$

from where the following first-integral is found [29]:

$$
\psi\left(x, y, v_{x}, v_{y}\right)=\left(\frac{x}{y}\right)^{2}+\xi^{2}=\left(\frac{x}{y}\right)^{2}+\left(x v_{y}-y v_{x}\right)^{2},
$$

which is the well-known Ermakov-Lewis invariant [27, 28, 40].

\subsection{The Pinney equation revisited}

We mentioned before the possibility of obtaining solutions of a given system from particular solutions of another related system. We next study a particular example which allows us to recover the results obtained by Pinney long time ago [21]. Consider the system of first-order differential equations:

$$
\begin{aligned}
& \dot{x}=v_{x}, \\
& \dot{y}=v_{y}, \\
& \dot{z}=v_{z}, \\
& \dot{v}_{x}=-\omega^{2}(t) x+\frac{k}{x^{3}}, \\
& \dot{v}_{y}=-\omega^{2}(t) y, \\
& \dot{v}_{z}=-\omega^{2}(t) z,
\end{aligned}
$$

which corresponds to the vector field

$$
X=v_{x} \frac{\partial}{\partial x}+v_{y} \frac{\partial}{\partial y}+v_{z} \frac{\partial}{\partial z}+\frac{k}{x^{3}} \frac{\partial}{\partial v_{x}}-\omega^{2}(t)\left(x \frac{\partial}{\partial v_{x}}+y \frac{\partial}{\partial v_{y}}+z \frac{\partial}{\partial v_{z}}\right) .
$$

The $t$-dependent vector field $X$ can be expressed as $X=N_{2}-\omega^{2}(t) N_{1}$ where $N_{1}$ and $N_{2}$ are

$$
N_{1}=y \frac{\partial}{\partial v_{y}}+x \frac{\partial}{\partial v_{x}}+z \frac{\partial}{\partial v_{z}}, \quad N_{2}=v_{x} \frac{\partial}{\partial x}+v_{y} \frac{\partial}{\partial y}+v_{z} \frac{\partial}{\partial z}+\frac{1}{x^{3}} \frac{\partial}{\partial v_{x}},
$$

These vector fields generate a 3-dimensional real Lie algebra with the vector field $N_{3}$ given by

$$
N_{3}=\frac{1}{2}\left(x \frac{\partial}{\partial x}+y \frac{\partial}{\partial y}+z \frac{\partial}{\partial z}-v_{x} \frac{\partial}{\partial v_{x}}-v_{y} \frac{\partial}{\partial v_{y}}-v_{z} \frac{\partial}{\partial v_{z}}\right) .
$$

In fact, they generate a Lie algebra isomorphic to $\mathfrak{s l}(2, \mathbb{R})$ because

$$
\left[N_{1}, N_{2}\right]=2 N_{3}, \quad\left[N_{3}, N_{1}\right]=N_{1}, \quad\left[N_{2}, N_{3}\right]=N_{2} .
$$


The dimension of the distribution generated by these fundamental vector fields is three and the manifold of the Lie system is of dimension six, then there are three time-independent integrals of motion which turn out to be the Ermakov invariant $I_{1}$ of the subsystem involving variables $x$ and $y$, the Ermakov invariant $I_{2}$ of the subsystem involving variables $x$ and $z$, and the Wronskian $W$ of the subsystem involving variables $y$ and $z$. They define a foliation with 3-dimensional leaves. This foliation can be used for obtaining a superposition rule.

The Ermakov invariants read as

$$
I_{1}=\frac{1}{2}\left(\left(y v_{x}-x v_{y}\right)^{2}+k\left(\frac{y}{x}\right)^{2}\right), \quad I_{2}=\frac{1}{2}\left(\left(x v_{z}-z v_{x}\right)^{2}+k\left(\frac{z}{x}\right)^{2}\right),
$$

where $I_{1}$ and $I_{2}$ are non-negative constants and the Wronskian $W$ is:

$$
W=y v_{z}-z v_{y} .
$$

We can obtain an explicit expression of $x$ in terms of $y, z$ and the three first integrals $I_{1}, I_{2}, W$ :

$$
x=\frac{\sqrt{2}}{|W|}\left(I_{2} y^{2}+I_{1} z^{2} \pm \sqrt{4 I_{1} I_{2}-k W^{2}} y z\right)^{1 / 2} .
$$

Remark that here $W$ is a constant fixed by the two independent particular solutions of the time-dependent harmonic oscillator $x_{1}(t)$ and $x_{2}(t)$ and only $I_{1}$ and $I_{2}$ play the role of constants in this superposition rule for the Milne-Pinney equation. This is not a surprising fact because the Milne-Pinney equation is a second-order differential equation. Note also that the values of $I_{1}$ and $I_{2}$ are non-negative constants but should be chosen such that $x(0)$ be real.

This can be interpreted, as pointed out by Pinney [21], as saying that there is a superposition rule allowing us to express the general solution of the Milne-Pinney equation in terms of two independent solutions of the corresponding harmonic oscillator with the same time-dependent angular frequency.

\subsection{A new superposition rule for the Pinney equation}

A new mixed superposition rule involving solutions of a Riccati for obtaining the general solution of Pinney equations is obtained in this section as a straightforward application of our development.

The $t$-dependent Riccati equation

$$
\frac{d x}{d t}=b_{0}(t)+b_{1}(t) x+b_{2}(t) x^{2} .
$$

has been studied in $[51,52]$ from the perspective of the theory of Lie systems. We follow here a very similar approach. From the geometric viewpoint the Riccati equation (16) can be considered as a differential equation determining the integral curves of the time-dependent vector field

$$
\Gamma=\left(b_{0}(t)+b_{1}(t) x+b_{2}(t) x^{2}\right) \frac{\partial}{\partial x},
$$

which is a linear combination with time-dependent coefficients of the three vector fields

$$
L_{1}=\frac{\partial}{\partial x}, \quad L_{2}=-x^{2} \frac{\partial}{\partial x}, \quad L_{3}=-x \frac{\partial}{\partial x},
$$

which close on a 3 -dimensional real Lie algebra, with defining relations

$$
\left[L_{1}, L_{2}\right]=2 L_{3}, \quad\left[L_{3}, L_{1}\right]=L_{1}, \quad\left[L_{2}, L_{3}\right]=L_{2},
$$

therefore isomorphic to $\mathfrak{s l}(2, \mathbb{R})$, because the commutation relations (17) are the same as $(12)$. 
The following particular case of Riccati equation:

$$
\frac{d x}{d t}=-\omega^{2}(t)-x^{2}
$$

is the equation for the integral curves of the $t$-dependent vector field $L=L_{2}-\omega^{2}(t) L_{1}$. Consequently, we can apply the procedure of the Section 4 and consider the following system of differential equations

$$
\begin{aligned}
& \dot{x}_{1}=-\omega^{2}(t)-x_{1}^{2}, \\
& \dot{x}_{2}=-\omega^{2}(t)-x_{2}^{2}, \\
& \dot{x}_{3}=-\omega^{2}(t)-x_{3}^{2}, \\
& \dot{x}=v, \\
& \dot{v}=-\omega^{2}(t) x+\frac{k}{x^{3}}
\end{aligned}
$$

described by a vector field in $\mathbb{R}^{3} \times \mathbb{T R}_{+}$, where $\left(x_{1}, x_{2}, x_{3}\right) \in \mathbb{R}^{3}, x \in \mathbb{R}_{+}$and $(x, v) \in T_{x} \mathbb{R}_{+}$. According to our general recipe, consider the following vector fields

$$
\begin{aligned}
& M_{1}=\frac{\partial}{\partial x_{1}}+\frac{\partial}{\partial x_{2}}+\frac{\partial}{\partial x_{3}}+x \frac{\partial}{\partial v}, \quad M_{2}=-x_{1}^{2} \frac{\partial}{\partial x_{1}}-x_{2}^{2} \frac{\partial}{\partial x_{2}}-x_{3}^{2} \frac{\partial}{\partial x_{3}}+v \frac{\partial}{\partial x}+\frac{k}{x^{3}} \frac{\partial}{\partial v}, \\
& M_{3}=-x_{1} \frac{\partial}{\partial x_{1}}-x_{2} \frac{\partial}{\partial x_{2}}-x_{3} \frac{\partial}{\partial x_{3}}+\frac{1}{2}\left(x \frac{\partial}{\partial x}-v \frac{\partial}{\partial v}\right),
\end{aligned}
$$

that, by construction, verify the same commutation relations as before, i.e.

$$
\left[M_{1}, M_{2}\right]=2 M_{3}, \quad\left[M_{3}, M_{1}\right]=M_{1}, \quad\left[M_{3}, M_{2}\right]=-M_{2},
$$

and the full system of differential equations can be understood as the system of differential equations for the determination of the integral curves of the $t$-dependent vector field $M=$ $M_{2}-\omega^{2}(t) M_{1}$. The dimension of the distribution associated to this Lie system is three in almost any point and then there exist two integrals of motion. As $2 M_{3}=\left[M_{1}, M_{2}\right]$, it is enough to find the simultaneously integrals of motion of $M_{1}$ and $M_{2}$, i.e. a function $F: \mathbb{R}^{5} \rightarrow \mathbb{R}$ such that $M_{1} F=M_{2} F=0$.

Let us first look for first-integrals independent of $x_{3}$, i.e. we suppose that $F$ depends just on $x_{1}, x_{2}, x$ and $v$. Using the method of characteristics, the condition $M_{1} F=0$ means that there is a function $\bar{F}: \mathbb{R}^{3} \rightarrow \mathbb{R}$ such that $F\left(x_{1}, x_{2}, x, v\right)=\bar{F}\left(I_{1}, I_{2}, I_{3}\right)$ with $I_{1}, I_{2}$ and $I_{3}$ given by

$$
I_{1}=x_{1}-x_{2}, \quad I_{2}=x_{2}-v / x, \quad I_{3}=x .
$$

Then, the condition $M_{2} \bar{F}=0$ reads in terms of the variables $I_{1}, I_{2}, I_{3}$ and $I_{4} \equiv v$ as

$$
I_{4}\left[-2 \frac{I_{1}}{I_{3}} \frac{\partial \bar{F}}{\partial I_{1}}-2 \frac{I_{2}}{I_{3}} \frac{\partial \bar{F}}{\partial I_{3}}+\frac{\partial \bar{F}}{\partial I_{3}}\right]+\left[\left(-I_{1}^{2}-2 I_{1} I_{2}\right) \frac{\partial \bar{F}}{\partial I_{1}}-\left(I_{2}^{2}+\frac{k}{I_{3}^{4}}\right) \frac{\partial \bar{F}}{\partial I_{2}}\right]=0 .
$$

Thus, the linear term in $I_{4}$ and the other one must vanish independently. The method of characteristics applied to the first term means that there exists a function $\widehat{F}: \mathbb{R}^{2} \rightarrow \mathbb{R}$ such that $\bar{F}\left(I_{1}, I_{2}, I_{3}\right)=\widehat{F}\left(K_{1}, K_{2}\right)$ where

$$
K_{1}=\frac{I_{1}}{I_{2}}, \quad K_{2}=I_{2} I_{3}^{2}
$$

Finally, taking into account the last result in $M_{2} \hat{F}=0$ we obtain

$$
\left(-K_{1}^{2}-K_{1}+\frac{k K_{1}}{K_{2}^{2}}\right) \frac{\partial \widehat{F}}{\partial K_{1}}-\left(K_{2}+\frac{k}{K_{2}}\right) \frac{\partial \widehat{F}}{\partial K_{2}}=0,
$$


and by means of the method of characteristics

$$
\frac{d K_{1}}{d K_{2}}=\frac{K_{1}^{2}+K_{1}-\frac{k K_{1}}{K_{2}^{2}}}{K_{2}+\frac{k}{K_{2}}} .
$$

Finally, taking into account the last result we obtain the first-integral

$$
C_{1}=K_{2}+\frac{k+K_{2}^{2}}{K_{1} K_{2}}
$$

that in terms of the initial variables reads as

$$
C_{1}=\left(x_{2}-\frac{v}{x}\right) x^{2}+\frac{k+\left(x_{2}-\frac{v}{x}\right)^{2} x^{4}}{\left(x_{1}-x_{2}\right) x^{2}} .
$$

If we repeat the last procedure but under the assumption that the integral does not depend on $x_{2}$ we obtain the following first-integral:

$$
C_{2}=\left(x_{3}-\frac{v}{x}\right) x^{2}+\frac{k+\left(x_{3}-\frac{v}{x}\right)^{2} x^{4}}{\left(x_{1}-x_{3}\right) x^{2}} .
$$

It is a long but straightforward computation to check out that both are integrals of $M_{1}, M_{2}$ and $M_{3}$. We can obtain now the general solution $x$ of (1) $x$ in terms of $x_{1}, x_{2}, x_{3}, C_{1}, C_{2}$ as

$$
x=\sqrt{\frac{\left(C_{1}\left(x_{1}-x_{2}\right)-C_{2}\left(x_{1}-x_{3}\right)\right)^{2}+k\left(x_{2}-x_{3}\right)^{2}}{\left(C_{2}-C_{1}\right)\left(x_{2}-x_{3}\right)\left(x_{2}-x_{1}\right)\left(x_{1}-x_{3}\right)}},
$$

where $C_{1}$ and $C_{2}$ are constants such that once $x_{1}(t), x_{2}(t)$ and $x_{3}(t)$ have been fixed they make $x(0)$ given by (18) to be real.

Thus we have obtained a new mixed superposition rule which allows us to express the general solution of the Pinney equation in terms of three solutions of Riccati equations, and of course two constants which determine each particular solution.

\section{Conclusions and outlook}

In this paper we have reviewed the theory developed in [6] in order to deal with a certain kind of systems second-order differential equations that can be studied as Lie systems, in particular with the Milne-Pinney equation and the Ermakov system. We have not only recovered in this way some known results about these differential equations, i.e. integrals of motion or superposition rules, but we have also been able to show new ones as a superposition rule for three solutions of a Riccati equation to give us the general solution of the Milne-Pinney equation.

New applications of this formalism to relate a Milne-Pinney equation with a frequency $\omega(t)$ with a $t$-dependent harmonic oscillator with different frequency $\omega^{\prime}(t)$ related with $\omega(t)$ by means of certain relations will be developed in forthcoming papers. In this way a new time-dependent superposition rule is obtained and by means of it we can express the solution of a Milne-Pinney equation with frequency $\omega(t)$ in terms of solutions of a time-dependent harmonic oscillator with frequency $\omega^{\prime}(t)$.

Another remarkable point to be studied is the extension of this formalism for studying some generalised Ermakov systems as those appearing for instance in [38] and [39].

\section{Acknowledgements}

Partial financial support by research projects MTM2006-10531 and E24/1 (DGA) are acknowledged. JdL also acknowledge a F.P.U. grant from Ministerio de Educación y Ciencia and a special grant from the Network of Mechanics, Geometry and Control. 


\section{References}

[1] Ermakov V.P., Second-order differential equations. Conditions of complete integrability, Univ. Isz. Kiev Series III 9 (1880), 1-25 (translation by A.O. Harin).

[2] Lie S., Vorlesungen über continuierliche Gruppen mit Geometrischen und anderen Anwendungen, edited and revised by G. Scheffers, Teubner, Leipzig, 1893.

[3] Ibragimov N.H., An ABC of group analysis, Novoe v Zhizni, Nauke, Tekhnike. Seriya Matematika, Kibernetika, no. 8, Znanie, Moscow, 1989 (in Russian).

Ibragimov N.H., Introduction to modern group analysis, Tau, Ufa, 2000 (revised edition in English).

[4] Ibragimov N.H., Elementary Lie group analysis and ordinary differential equations, J. Wiley, Chichester, 1999.

[5] Winternitz P., Lie groups and solutions of nonlinear differential equations, in Nonlinear Phenomena, Editor K.B. Wolf, Lecture Notes in Physics, Vol. 189, Springer-Verlag, New York, 1983, 263-305.

[6] Cariñena J.F., Grabowski J., Marmo G., Lie-Scheffers systems: a geometric approach, Bibliopolis, Napoli, 2000.

[7] Cariñena J.F., Grabowski J., Ramos A., Reduction of time-dependent systems admitting a superposition principle, Acta Appl. Math. 66 (2001), 67-87.

[8] Cariñena J.F., Grabowski J., Marmo G., Some applications in physics of differential equation systems admitting a superposition rule, Rep. Math. Phys. 48 (2001), 47-58.

[9] Anderson R.L., A nonlinear superposition principle admitted by coupled Riccati equations of the projective type, Lett. Math. Phys. 4 (1980), 1-7.

[10] Harnad J., Winternitz P., Anderson R.L., Superposition principles for matrix Riccati equations, J. Math. Phys. 24 (1983), 1062-1072.

[11] del Olmo M.A., Rodríguez M.A., Winternitz P., Simple subgroups of simple Lie groups and nonlinear differential equations with superposition principles, J. Math. Phys. 27 (1986), 14-23.

[12] Kevrekidis P.G., Drossinos Y., Nonlinearity from linearity: the Ermakov-Pinney equation revisited, Math. Comput. Simulation 74 (2007), 196-202.

[13] Leach P.G.L., Karasu A., Nucci M.C., Andriopoulos K., Ermakov's superintegrable toy and nonlocal symmetries, SIGMA 1 (2005), 018, 15 pages, nlin.SI/0511055.

[14] del Olmo M.A., Rodríguez M.A., Winternitz P., Superposition formulas for rectangular matrix Riccati equations, J. Math. Phys. 28 (1987), 530-535.

[15] Cariñena J.F., Ramos A., Riccati equation, factorization method and shape invariance, Rev. Math. Phys. 12 (2000), 1279-1304, math-ph/9910020.

[16] Cariñena J.F., Ramos A., A new geometric approach to Lie systems and physical applications, Acta Appl. Math. 70 (2002), 43-69, math-ph/0110023.

[17] Cariñena J.F., Marmo G., Nasarre J., The nonlinear superposition principle and the Wei-Norman method, Internat. J. Modern Phys. A 13 (1998), 3601-3627, physics/9802041.

[18] Cariñena J.F., Grabowski J., Marmo G., Superposition rules, Lie theorem and partial differential equations, Rep. Math. Phys. 60 (2007), 237-258 math-ph/0610013.

[19] Cariñena J.F., de Lucas J., Rañada M.F., Nonlinear superposition rules and Ermakov systems, in Differential Geometric Methods in Mechanics and Field Theory, Editors F. Cantrijn, M. Crampin and B. Langerock, Academia Press, 2007, 15-33.

[20] Milne W.E., The numerical determination of characteristic numbers, Phys. Rev. 35 (1930), 863-67.

[21] Pinney E., The nonlinear differential equation $y^{\prime \prime}+p(x) y+c y^{-3}=0$, Proc. Amer. Math. Soc. 1 (1950), 681.

[22] Hawkins R.M., Lidsey J.E., Ermakov-Pinney equation in scalar field cosmologies, Phys. Rev. D 66 (2002), 023523, 8 pages, astro-ph/0112139.

[23] Lidsey L.E., Cosmic dynamics of Bose-Einstein condensates, Classical Quantum Gravity 21 (2004), 777-785, gr-qc/0307037.

[24] Haas F., Anisotropic Bose-Einstein condensates and completely integrable dynamical systems, Phys. Rev. A 65 (2002), 033603, 6 pages, cond-mat/0211353.

[25] Fernández Guasti M., Moya-Cessa H., Amplitude and phase representation of quantum invariants for the time-dependent harmonic osicllator, Phys. Rev. A 67 (2003), 063803, 5 pages, quant-ph/0212073. 
[26] Gauthier S., An exact invariant for the time dependent double well anharmonic oscillators: Lie theory and quasi-invariance groups, J. Phys. A: Math. Gen. 17 (1984), 2633-2639.

[27] Ray J.R., Reid J.L., More exact invariants for the time-dependent harmonic oscillator, Phys. Lett. A 71 (1979), 317-318.

[28] Dhara A.K., Lawande S.V., Time-dependent invariants and the Feynman propagator, Phys. Rev. A 30 (1984), 560-567.

[29] Lewis H.R., Classical and quantum systems with time-dependent harmonic-oscillator-type Hamiltonians, Phys. Rev. Lett. 18 (1967), 510-512.

[30] Reid J.L., Ray J.R., Ermakov systems, Noether's theorem and the Sarlet-Bahar method, Lett. Math. Phys. 4 (1980), 235-240.

[31] Cerveró J.M., Lejarreta J.D., Ermakov Hamiltonians, Phys. Lett. A 156 (1991), 201-205.

[32] Sarlet W., Exact invariants for time-dependent Hamiltonian systems with one degree of freedom, J. Phys. A: Math. Gen. 11 (1978), 843-854.

[33] Govinder K.S., Athorne C., Leach P.G.L., The algebraic structure of generalized Ermakov systems in three dimensions, J. Phys. A: Math. Gen. 26 (1993), 4035-4046.

[34] Athorne C., Rogers C., Ramgulam U., Osbaldestin A., On linearization of the Ermakov system, Phys. Lett. A 143 (1990), 207-212.

[35] Sarlet W., Cantrijn F., A generalization of the nonlinear superposition idea for Ermakov systems, Phys. Lett. A 88 (1982), 383-387.

[36] Reid J.L., Ray J.R., Ermakov systems, nonlinear superposition and solutions of nonlinear equations of motion, J. Math. Phys. 21 (1980), 1583-1587.

[37] Govinder K.S., Leach P.G.L., Ermakov systems: a group theoretic approach, Phys. Lett. A 186 (1994), 391-395.

[38] Athorne C., Projective lifts and generalised Ermakov and Bernoulli systems, J. Math. Anal. Appl. 233 (1999), 552-563.

[39] Rogers C., Schief W.K., Bassom A., Ermakov systems with arbitrary order, dimension. Structure and linearisation, J. Phys. A: Math. Gen. 29 (1996), 903-911.

[40] Leach P.G.L., Generalized Ermakov systems, Phys. Lett. A 158 (1991), 102-106.

[41] Sarlet W., Further generalization of Ray-Reid systems, Phys. Lett. A 82 (1981), 161-164.

[42] Calogero F., Solution of a three body problem in one dimension, J. Math. Phys. 10 (1969), 2191-2196.

[43] Perelomov A.M., Integrable systems of classical mechanics and Lie algebras, Birkhäuser Verlag, Basel, 1990.

[44] Chalykh O.A., Vesselov A.P., A remark on rational isochronous potentials, J. Nonlinear Math. Phys. 12 (2005), suppl. 1, 179-183, math-ph/0409062.

[45] Asorey M., Cariñena J.F., Marmo G., Perelomov A.M., Isoperiodic classical systems and their quantum counterparts, Ann. Phys. 322 (2007), 1444-1465, arXiv:0707.4465.

[46] Cariñena J.F., Perelomov A.M., Rañada M.F., Isochronous classical systems and quantum systems with equally spaced spectra, in Particles and Fields: Classical and Quantum, J. Phys. Conf. Ser. 87 (2007), 012007, 14 pages.

[47] Leach P.G.L., Karasu A., The Lie algebra $s l(2, \mathbb{R})$ and so-called Kepler-Ermakov systems, J. Nonlinear Math. Phys. 11 (2004), 269-275.

[48] Karasu A., Yildrim H., On the Lie symmetries of the Kepler-Ermakov systems, J. Nonlinear Math. Phys. 9 (2002), 475-482, math-ph/0306037.

[49] Ray J.R., Reid J.L., Exact time-dependent invariants for N-dimensional systems, Phys. Lett. A 74 (1979), 23-25.

[50] Ray J.R., Invariants for nonlinear equations of motion, Progr. Theoret. Phys. 65 (1981), 877-882.

[51] Cariñena J.F., Ramos A., Integrability of the Riccati equation from a group theoretical viewpoint, Internat. J. Modern Phys. A 14 (1999), 1935-1951, math-ph/9810005.

[52] Cariñena J.F., de Lucas J., Ramos A., A geometric approach to integrability conditions for Riccati equations, Electron. J. Differential Equations 2007 (2007), 122, 14 pages. 